\title{
Calibration of Simulation Models for Ultrasonic Transducers Based on Implicit Calibration
}

\author{
Johan E. Carlson*, Jesper Martinsson ${ }^{\dagger}$, Fredrik Hägglund ${ }^{\dagger}$, Amin Saremi* $^{*}$ \\ *Div. of Systems and Interaction, Dept. of Computer Science and Electrical Engineering \\ Luleå University of Technology, SE-971 87 Luleå, Sweden \\ ${ }^{\dagger}$ EISLAB, Dept. of Computer Science and Electrical Engineering \\ Luleå University of Technology \\ SE-971 87 Luleå, Sweden \\ *Email: Johan.Carlson@1tu.se
}

\begin{abstract}
There are numerous software packages available for modeling of the sound pressure fields emitted by ultrasound transducers and transducer arrays. Accurate modeling of a realworld transducer requires knowledge of several parameters that are generally not known. This paper presents an estimation principle that can be used to calibrate such models, based on measurements of the sound field. The model separates the problem into estimation of the transducer's electro-mechanical impulse response and its spatial impulse response. The latter being what the software packages generally model.

We demonstrate the principle with measurements of the sound field from a non-focused $5 \mathrm{MHz}$ transducer. The results show that the modeled sound field agrees well with measurements.

Index Terms-Implicit calibration, transducer modeling, ultrasound measurements.
\end{abstract}

\section{INTRODUCTION}

The ability to accurately model the sound field emitted by a transducer or a transducer array is valuable when developing any kind of new measurement technology based on ultrasound. There are several software packages available for this purpose, whereof two MATLAB toolboxes stand out as the ones being most frequently used. The most commonly referenced toolbox is Field II by Jensen [1], [2], which has been available for several years running on several platforms.

Another powerful toolbox is the DREAM toolbox, introduced by Lingvall and Piwakowski [3]. It provides functionality similar to that of Field II, but all processing is done directly in the discrete-time domain. Also, it supports multi-core processors, which significantly speeds up calculations on a modern computer. As of 2007 the DREAM toolbox is available as open source, meaning that the users can modify functions and also contribute to the development of the toolbox.

The DREAM toolbox is based on the principles outlined by Piwakowski in [4], [5] for calculation of spatial impulse responses of transducers and arrays of arbitrary geometries.

The toolboxes provide the possibility of simulating the behavior of an ultrasound measurement system, but they lack one feature, which is addressed in this paper. When setting up a simulation, each transducer model is described by a number of parameters that are assumed to be known. One also generally assumes that the electro-mechanical impulse response is known (i.e. the shape of the acoustic pulse, at the transducer surface). In reality, neither of these are known.

In this paper we present a method based on the principle of implicit calibration [6], [7] for simultaneous estimation of the electro-mechanical impulse response and the transducer simulation parameters. This could prove useful if we want to design a simulation model of a transducer actually available in the lab.

We will start by formalizing the system model for the general case and then describe how the principle of implicit calibration can be used to identify the model. As a proof of concept we then show with experiments how this can be applied to the case of a simple circular transducer.

\section{THEORY}

In this section we start by setting up a linear systems description of the transducer model, from the electro-mechanical impulse response of the transducer, via the spatial impulse response (SIR) of the transducer and the propagation medium, to the observed sound pressure waveform.

\section{A. System model}

Assume that the ultrasound transducer in Fig. 1 is excited by an impulse at time $t=0$. The transducer's electro-mechanical impulse response, $h_{e}(t)$ will then cause the surface to vibrate, generating a pressure field.
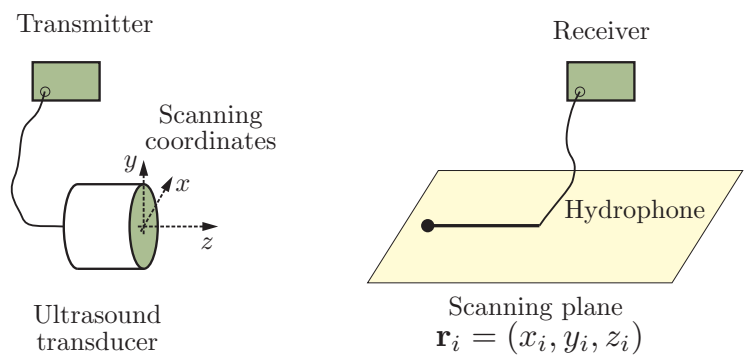

Fig. 1. Experimental setup with a circular transducer and a calibrated receiving hydrophone.

Under the assumption that linear acoustics apply then, at the position $\left(x_{i}, y_{i}, z_{i}\right)$ of the hydrophone, the received signal, 


$$
\begin{aligned}
& \tilde{u}_{i}\left(x_{i}, y_{i}, z_{i}, t\right) \text { will be } \\
& \qquad \begin{aligned}
& \tilde{u}_{i}\left(x_{i}, y_{i}, z_{i}, t\right)= \\
& \tilde{h}_{e}(t) * \tilde{h}_{S I R}\left(x_{i}, y_{i}, z_{i}, t ; \boldsymbol{\theta}\right) * \tilde{h}_{M}\left(x_{i}, y_{i}, z_{i}, t\right),
\end{aligned}
\end{aligned}
$$

where $\tilde{h}_{S I R}\left(x_{i}, y_{i}, z_{i}, t, \boldsymbol{\theta}\right)$ is the spatial impulse response between the transducer surface and the observation point, and $\tilde{h}_{M}\left(x_{i}, y_{i}, z_{i}, t\right)$ is the impulse response of the medium between the transducer and the observation point. The * denotes convolution in the time domain. The $\tilde{\text { symbol is used }}$ to denote time-domain versions of the quantities. By using a calibrated hydrophone to measure the sound pressure field, we impose the assumption that the hydrophone itself does not affect the pulse. Furthermore, we require that the spatial impulse responses can be calculated using a numerical model, given some parameters in the vector $\boldsymbol{\theta}$.

For notational simplicity, we can replace the observation point coordinates $\left(x_{i}, y_{i}, z_{i}\right)$ with the vector $\mathbf{r}_{i}$. Eq. (1) now simplifies to

$$
\tilde{u}_{i}\left(\mathbf{r}_{i}, t\right)=\tilde{h}_{e}(t) * \tilde{h}_{S I R}\left(\mathbf{r}_{i}, t ; \boldsymbol{\theta}\right) * \tilde{h}_{M}\left(\mathbf{r}_{i}, t\right) .
$$

Taking the Fourier transform on both sides of Eq. (2), we obtain the analogous frequency domain representation as

$$
u_{i}(\omega)=h_{e}(\omega) h_{S I R}\left(\omega, \mathbf{r}_{i} ; \boldsymbol{\theta}\right) h_{M}\left(\omega, \mathbf{r}_{i}\right) .
$$

If the propagation medium is pure water, and all observation points are in the far field of the transducer, we can (in practice) approximate the transfer function of the medium as just a scalar attenuation that depends on the propagation distance, $\left\|\mathbf{r}_{i}\right\|=\sqrt{x_{i}^{2}+y_{i}^{2}+z_{i}^{2}}$, and the frequency of the transducer. The model then becomes

$$
u_{i}(\omega)=h_{e}(\omega) h_{S I R}\left(\omega, \mathbf{r}_{i} ; \boldsymbol{\theta}\right) e^{-\alpha\left\|\mathbf{r}_{i}\right\|},
$$

where $\alpha$ is the absorption coefficient of water. We can choose to either estimate $\alpha$ or assume that it is known, given the temperature and frequency of the transducer. In the example presented in the next section, we assume it is known from theory [8].

So, recall the signal model:

$$
u_{i}=h_{e} h_{S I R}\left(\mathbf{r}_{i} ; \boldsymbol{\theta}\right) e^{-\alpha\left\|\mathbf{r}_{i}\right\|},
$$

which is the same as in Eq. (4), but with the $\omega$ denoting frequency was dropped for notational simplicity. In this configuration, $h_{e}$ is unknown but constant, $h_{S I R}\left(\mathbf{r}_{i} ; \boldsymbol{\theta}\right)$ can be calculated using the DREAM toolbox, but the parameter vector $\boldsymbol{\theta}$ is unknown.

Since we are going to use sampled versions of all quantities, we rewrite the Eq. (5) in matrix notation as

$$
\mathbf{u}_{i}=\mathbf{H}_{i} \mathbf{h}_{e},
$$

where $\mathbf{u}_{i}$ and $\mathbf{h}_{e}$ are vectors containing the frequency-domain versions of their sampled continuous-time counterparts, and where

$$
\mathbf{H}_{i}=\operatorname{diag}\left[\begin{array}{c}
h_{S I R}\left(\omega_{1}, \mathbf{r}_{i} ; \boldsymbol{\theta}\right) e^{-\alpha\left\|\mathbf{r}_{i}\right\|} \\
h_{S I R}\left(\omega_{2}, \mathbf{r}_{i} ; \boldsymbol{\theta}\right) e^{-\alpha\left\|\mathbf{r}_{i}\right\|} \\
\vdots \\
h_{S I R}\left(\omega_{N}, \mathbf{r}_{i} ; \boldsymbol{\theta}\right) e^{-\alpha\left\|\mathbf{r}_{i}\right\|}
\end{array}\right]
$$

i.e. a diagonal matrix with the diagonal elements as in Eq. (7).

Now, assume we measure the sound field at $M$ different positions, given by the set $\left\{\mathbf{r}_{i}\right\}$, for $i=1,2, \ldots, M$. Then define

$$
\mathbf{u}=\left[\begin{array}{c}
\mathbf{u}_{1} \\
\mathbf{u}_{2} \\
\vdots \\
\mathbf{u}_{M}
\end{array}\right], \quad \mathbf{H}_{\boldsymbol{\theta}}=\left[\begin{array}{c}
\mathbf{H}_{1} \\
\mathbf{H}_{2} \\
\vdots \\
\mathbf{H}_{M}
\end{array}\right]
$$

Using the definitions in Eq. (8), we then obtain an expression for the entire system, as

$$
\mathbf{u}=\mathbf{H}_{\boldsymbol{\theta}} \mathbf{h}_{e} .
$$

The task is to use $\mathbf{u}$ to simultaneously estimate the unknown parameters $\boldsymbol{\theta}$ of the simulation model and the electromechanical impulse response $\mathbf{h}_{e}$.

\section{B. Implicit calibration}

The system model described in the previous section contains two unknown parts. The first is the electro-mechanical impulse response of the transducer, i.e. the shape of the transmitted ultrasound pulse at the transducer surface. This is independent of where in the sample volume we measure the pulse, i.e. independent of $\mathbf{r}_{i}$. The second is the spatial impulse response (SIR) of the transducer, which varies over the measurement volume. Given a set of parameters $\boldsymbol{\theta}$, the SIR can be calculated using numerical models. For a given model of the SIR, a leastsquares estimate of the electro-mechanical impulse response is readily available (e.g. by Eq. (10).

So, by collecting measurements at a number of different locations in front of the transducer, we can iteratively estimate the two unknown impulse responses by separating the nonlinear optimization (estimation of $\boldsymbol{\theta}$ ) from the linear regression (estimation of $\mathbf{h}_{e}$ ). The resulting algorithm is an iterative procedure known as implicit calibration, since the calibration (or regression) giving $\mathbf{h}_{e}$ is done inside the non-linear optimization.

The procedure is as follows:

1) Set the current iteration $k=0$

2) Given the physical properties of the setup, assign some starting guess of the parameter vector, denoted $\boldsymbol{\theta}^{(k)}$

3) Calculate the SIRs and the medium impulse response, given $\boldsymbol{\theta}^{(k)}$. This gives $\mathbf{H}_{\boldsymbol{\theta}}^{(k)}$ according to Eqs. (9) and (8).

4) Estimate the electro-mechanical impulse response of the $k$ :th iteration, $\mathbf{h}_{e}^{(k)}$ using some regression technique, e.g.

$$
\widehat{\mathbf{h}}_{e}^{(k)}=\left(\mathbf{H}_{\boldsymbol{\theta}}^{(k) T} \mathbf{H}_{\boldsymbol{\theta}}^{(k)}\right)^{-1} \mathbf{H}_{\boldsymbol{\theta}}^{(k) T} \mathbf{u} \text {. }
$$

5) Use the estimated electro-mechanical impulse response $\widehat{\mathbf{h}}^{(k)}$ and the calculated SIRs $\mathbf{H}_{\boldsymbol{\theta}}^{(k)}$ to estimate the sound pressure field

$$
\widehat{\mathbf{u}}=\mathbf{H}_{\boldsymbol{\theta}}^{(k)} \widehat{\mathbf{h}}_{e}^{(k)} \text {. }
$$

6) Determine the residual error between the modeled and observed sound pressure fields

$$
\mathbf{e}=\mathbf{u}-\widehat{\mathbf{u}}
$$


7) Update the estimate of $\boldsymbol{\theta}$, using some numerical optimization technique (minimizing for example $\|\mathbf{e}\|^{2}$ ), yielding $\boldsymbol{\theta}^{(k+1)}$. In this paper, the Nelder-Mead simplex search was used [9].

8) Increase $k$ by one and iterate steps 3-7 until some criterion of convergence is met.

\section{EXPERIMENTAL RESULTS}

In principle, the methodology described in the previous section can be used to estimate parameters of transducers of arbitrary geometries, as long as a numerical model which is parameterized using a reasonable number of parameters is available. However, as a demonstration of the principle, we will present results only for a simple circular transducer. This will give the reader some insight into the modeling, without getting lost in detailed discussions on specific transducer models. Also, in some cases the simple circular transducer can actually be described with analytical expressions, which further increases the possibilities for the user to evaluate the algorithm proposed here.

For the experiments we used a unfocused circular transducer with a center frequency of $5 \mathrm{MHz}$, (Panametrics, model V3428). The sound field was measured using a calibrated wideband hydrophone (FORCE Technology, model MHA9150). The transducer was mounted on a step motor controlled support, so that it could move relative to the hydrophone (see Fig. 1).

The sound field was measured in a plane of $31 \times 40$ points in the far field of the transducer, using a digitizing oscilloscope (GaGe CompuScope 12400), with a sampling frequency of $200 \mathrm{MHz}$ and a 12 bit resolution. The measured pulses were then stored in a vector $\mathbf{u}$ according to Eq. (8). The normalized energy of the pulses measured at each point is shown (in $\mathrm{dB}$ ) in Fig. 2. The yellow markers show which points were used for the calibration of the model. The choice of calibration points is arbitrary, but since the simulation model assumes circular symmetry, there is no reason to sample the grid uniformly in the entire plane. The number of points is also arbitrary, but it is a trade-off between performance and computational complexity and memory requirements. In this example we used the 28 points as indicated in Fig. 2.

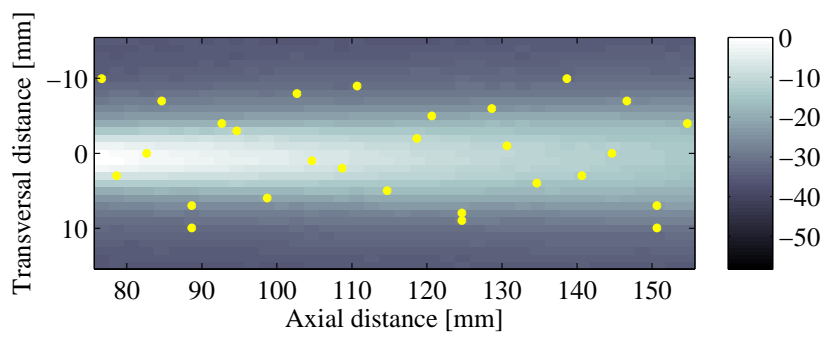

Fig. 2. Normalized energy (in $\mathrm{dB}$ ) of the measured pulses in a plane $\mathrm{i}$ front of the transducer. The circular markers indicate the location of the pulses used for the calibration.

The algorithm was initiated by setting the starting parameters of the model (in this case only the effective radius of

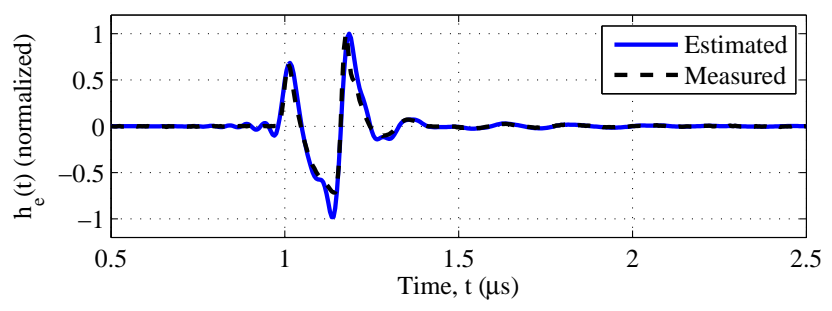

Fig. 3. The estimated (solid) electro-acoustic impulse response $h_{e}(t)$ of the transducer and a pulse measured along the center axis of the transducer (dashed). Both pulses are normalized in amplitude.

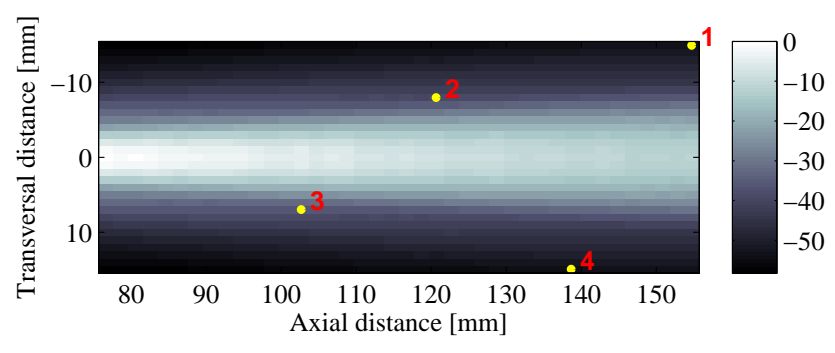

Fig. 4. Modeled pressure sound pressure energy. The markers represents locations of pulses 1-4 shown in Fig. 5.

the transducer) measured with a caliper to be $\boldsymbol{\theta} \approx 3 \mathrm{~mm}$. The absorption coefficient of the medium (water) was determined by measuring the average temperature in the water tank during the measurements $\left(T=22.16{ }^{\circ} \mathrm{C}\right)$ and using Eq. (10) of [8]. The resulting absorption coefficient was $\alpha=0.575 \mathrm{~Np} / \mathrm{m}$. After the calibration we obtained a value for the effective radius of the transducer, $r=2.353 \mathrm{~mm}$. The resulting estimate of the electro-mechanical impulse response is shown in Fig. 3. Since the electro-mechanical impulse response is not possible to measure separately, no fair comparison can be given to observed data. However, if we measure the sound field along the center line of the transducer, we see that the estimated impulse response agrees well with what we can expect from theory.

Using the estimated value of the transducer radius $r$ and the electro-mechanical impulse response (Fig. 3), we then simulated the sound pressure field. The resulting sound pressure energy is shown in Fig. 4. Note that the color scale is set to the same as in Fig. 2, but because of the noise floor in the measured sound pressure field, the figures appear to differ more than they really do. To illustrate this, the actual pulses, both measured and modeled are shown in Fig. 5, for the points marked and labeled in Fig. 4.

\section{Discussion}

In this paper we only used a model of a simple circular transducer. For the transducer we used here this seems to be enough, but in other situations, this model may be too simple to model the behavior of the real transducer. Extending the algorithm to use for example an annular array model, with varying apodization functions [3], all we need to do is to include the parameters of this in the vector $\boldsymbol{\theta}$. As long as 

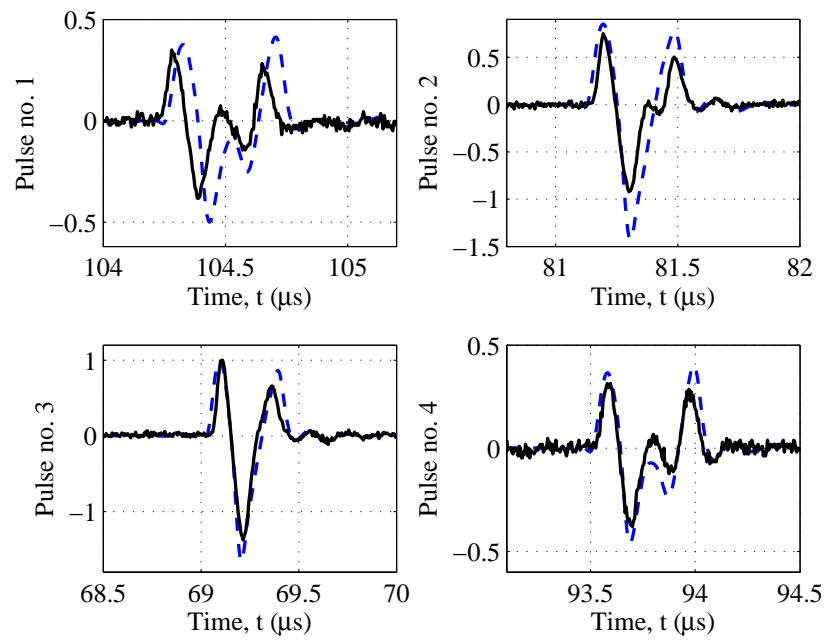

Fig. 5. Example of measured (solid lines) and modeled (dashed lines) pulses, as marked in Fig. 4.

the variations in the sound field are large enough, we will still be able to estimate such a model.

Similarly, using this approach to model the response of a real-world transducer array should be straightforward, as long as the simulation toolbox at hand supports array impulse response modeling, which is the case for both DREAM and Field II.

The most complicated issue in matching sound field measurements to the simulation model, which is crucial for the implicit calibration algorithm to work, is that we need to know exactly at which points in the measurement volume we actually acquired the data. Achieving sufficient accuracy in the positioning of the transducer relative to the hydrophone just by building carefully and using precise step motor might be enough. However, in our case we needed some automated adjustment scheme so, under the assumption that the sound pressure field was circular symmetric, we programmed the step motor to search for the amplitude maximum at a plane in the far field, parallel to the transducer surface. Once this automatic centering of the transducer was complete, we used the knowledge of the speed of sound in the water tank to estimate the distance between the transducer and the hydrophone. The remainder of the measurement could then be performed relying solely on the precision of the step motor positioning of the transducer. It might be worth noting here that this experimental issue was the most difficult and critical step in order to make the calibration work. That said, the slight phase difference between the measured and modeled pulses in Fig. 5 (Pulse no. 1) is likely to be caused by a small error in the positioning.

Another reason for the slight mismatch between the modeled and measured sound fields is that the transducer model has only one parameter (the radius). This means we have limited degrees of freedom in adapting the model. Moving to a more complicated transducer description is likely to improve the results.

\section{Conclusions}

In this paper we have set up a system model for the propagation of sound from a transducer to a position in a measurement volume in front of the transducer. We showed that this system can be divided into a constant electro-mechanical impulse response (unknown) and spatial impulse response, described by a parametric numerical model (unknown parameters). We then outlined a strategy for how to simultaneously estimate the two unknowns by probing the sound pressure field at a number of points in the far field of the transducer with a calibrated hydrophone. The estimation algorithm is an iterative solution based on separating the parameter estimation step (transducer model) from a linear regression step (the electro-mechanical impulse response), using the principle of implicit calibration.

We then demonstrated the algorithm using measurements of the sound field emitted by a simple circular transducer. The calibration procedure results in a simulation model that mimics the behavior of the real-world system in a satisfactory manner. We then discussed reasons for the deviations between the modeled and measured sound fields.

By being able to calibrate a simulation model to a realworld transducer, we are now able to simulate measurement setups and signal processing algorithms based on simulated data that are much more realistic than before.

\section{ACKNOWLEDGMENTS}

The authors would like to express their sincerest gratitude towards Assoc. Prof. Veli-Matti Taavitsainen at EVTEK University of Applied Sciences for valuable comments and discussions regarding the principles of implicit calibration. The authors would also like to thank Dr. Fredrik Lingvall at the University of Oslo for his help on issues regarding the DREAM toolbox.

\section{REFERENCES}

[1] J. A. Jensen, "Field: A program for simulating ultrasound systems," Medical \& Biological Engineering \& Computing, vol. Part 1, no. Supplement 1, pp. 351-353, 1996.

[2] J. A. Jensen and N. B. Svendsen, "Calculation of pressure fields from arbitrarily shaped, apodized, and excited ultrasound transducers," IEEE Trans. Ultrason., Ferroelec., Freq. Contr., vol. 39, pp. 262-267, 1992.

[3] F. Lingvall, "The DREAM toolbox," http://www.signal.uu.se/Toolbox/dream/, Visited: July 4, 2008.

[4] B. Piwakowski and B. Delannoy, "Method for computing spatial impulse response: Time-domain approach," J. Acoust. Soc. Am., vol. 86, pp. 2422 2432, 1989.

[5] B. Piwakowski and K. Sbai, "A new approach to calculate the field radiated from arbitrarily structured transducer arrays," IEEE Trans. Ultrason., Ferroelec., and Freq. Contr., vol. 46, pp. 422-440, 1999.

[6] V.-M. Taavitsainen and H. Haario, "Rapid estimation of kinetics by implicit calibration. I," J. Chemometrics, vol. 15, pp. 215-239, 2001.

[7] V.-M. Taavitsainen, H. Haario, and M. Laine, "Rapid estimation of kinetics by implicit calibration. II," J. Chemometrics, vol. 17, pp. 140$150,2002$.

[8] F. H. Fisher and V. P. Simmons, "Sound absorption in sea water," $J$. Acoust. Soc. Am., vol. 62, no. 3, pp. 558-564, 1977.

[9] W. Murray, Ed., Numerical Methods for Unconstrained Optmization. New York: Academic Press, 1972. 